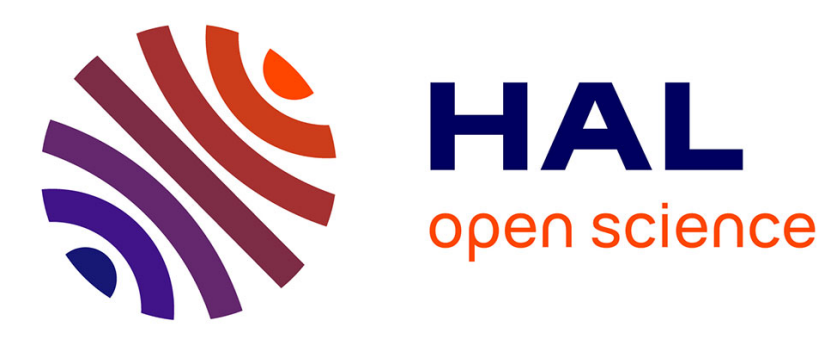

\title{
Théorie de l'ordre et syntactique chez Jules Bourgoin
}

Jenny Boucard, Christophe Eckes

\section{To cite this version:}

Jenny Boucard, Christophe Eckes. Théorie de l'ordre et syntactique chez Jules Bourgoin. Bideault, M. \& Thibault, E. \& Volait, M. De l'Orient à la mathématique de l'ornement: Jules Bourgoin (18381908), Picard, pp.281 - 298, 2015, 10.4000/books.inha.7035 . halshs-01351720

\section{HAL Id: halshs-01351720 \\ https://shs.hal.science/halshs-01351720}

Submitted on 10 Apr 2021

HAL is a multi-disciplinary open access archive for the deposit and dissemination of scientific research documents, whether they are published or not. The documents may come from teaching and research institutions in France or abroad, or from public or private research centers.
L'archive ouverte pluridisciplinaire HAL, est destinée au dépôt et à la diffusion de documents scientifiques de niveau recherche, publiés ou non, émanant des établissements d'enseignement et de recherche français ou étrangers, des laboratoires publics ou privés. 
De l'Orient à la mathématique de l'ornement. Jules

Bourgoin (1838-1908)

Publications de l'Institut national d'histoire de l'art

\section{Théorie de l'ordre et syntactique chez Jules Bourgoin}

\section{Jenny Boucard et Christophe Eckes}

DOI : 10.4000/books.inha.7035

Éditeur : Publications de l'Institut national d'histoire de l'art, Picard

Lieu d'édition : Paris

Année d'édition : 2015

Date de mise en ligne : 5 décembre 2017

Collection : InVisu

ISBN électronique : 9782917902813

\section{Oanctition ebooks}

http://books.openedition.org

Édition imprimée

Date de publication : 1 octobre 2015

Ce document vous est offert par Université de Nantes

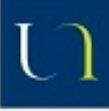

UNIVERSITÉ DE NANTES

\section{Référence électronique}

BOUCARD, Jenny; ECKES, Christophe. Théorie de l'ordre et syntactique chez Jules Bourgoin In : De l'Orient à la mathématique de l'ornement. Jules Bourgoin (1838-1908) [en ligne]. Paris : Publications de I'Institut national d'histoire de l'art, 2015 (généré le 10 avril 2021). Disponible sur Internet : <http:// books.openedition.org/inha/7035>. ISBN : 9782917902813. DOI : https://doi.org/10.4000/books.inha. 7035.

Ce document a été généré automatiquement le 10 avril 2021. 


\title{
Théorie de l'ordre et syntactique chez Jules Bourgoin
}

\author{
Jenny Boucard et Christophe Eckes
}

1 La référence à Antoine-Augustin Cournot est omniprésente dans les ouvrages et les notes de lecture de Bourgoin ${ }^{1}$. Dans l'introduction aux Études architectoniques et graphiques, il dit s'être imprégné de son œuvre lors d'une première mission «dans l'Orient musulman ${ }^{2}$ ", soit entre 1863 et 1866 . En outre, Cournot est une source centrale pour l'élaboration de la Théorie de l'ornement dont le titre initial, «L'ordre et la forme », est emprunté au premier chapitre de son Traité de l'enchaînement des idées ${ }^{3}$. Bourgoin affirme même avoir communiqué son manuscrit à Cournot en $1868^{4}$.

Dès cet ouvrage, Bourgoin s'intéresse à trois aspects de la notion d'ordre telle qu'elle est développée par Cournot: tout d'abord les correspondances entre arithmétique et géométrie d'une part, algèbre et géométrie d'autre part, thématisées par Cournot en $1847^{5}$, avec en toile de fond la référence au mathématicien Louis Poinsot; ensuite une classification des sciences formelles fondée sur «l'ordre et la forme »; enfin le projet d'une "syntactique», c'est-à-dire d'une théorie des combinaisons, dont cournot précise les contours dès $1843^{6}$. Ces emprunts permettent à Bourgoin de clarifier les rapports entre les sciences mathématiques et les arts d'ornement. Il aboutit ce faisant à un système théorique, tout en maintenant une nette distance entre ces deux domaines: ni le dessin d'ornement ni la graphique - qu'il développera à la fin de sa carrière - ne doivent être conçus dans le prolongement de la géométrie élémentaire. Il s'appuie par ailleurs sur ces deux notions d'ordre et de forme pour caractériser des types d'ornement, les classer et les combiner, dans le cadre d'une grammaire de l'ornement, ce que l'on peut rapprocher de la syntactique cournotienne.

3 Les sources de Bourgoin sur l'ordre, la forme et les combinaisons rayonnent principalement à partir de cette référence première, centrale et récurrente à Cournot. Par son intermédiaire, Bourgoin prend connaissance de certains travaux du mathématicien Louis Poinsot, qui développe dès le premier XIXe siècle une "théorie de l'ordre » relevant exclusivement des mathématiques. L'artiste étudie par ailleurs des mémoires de Johann-Heinrich Lambert et d'Alexandre-Théophile Vandermonde. 
Comprendre comment Bourgoin s'approprie puis modèle pour ses propres usages la thématique de l'ordre développée par ces différents auteurs, signifie montrer que le thème de l'ordre est intimement relié, chez Poinsot, Cournot et Bourgoin, à l'étude de configurations géométriques centrées sur le cercle et les polygones réguliers ; puis voir ensuite comment Bourgoin complète la classification des sciences logicomathématiques établie par Cournot en 1861, en y intégrant l'instrument du dessin ; par ailleurs, mesurer jusqu'à quel point Bourgoin est marqué par la syntactique de Cournot et enfin, étudier quelques applications de la théorie de l'ordre dans l'art ornemental en nous référant aux frises et aux figures textiles.

\section{Figures polygonales et ordre}

5 La thématique des polygones traverse l'œuvre de Bourgoin, d'un point de vue tant esthétique, formel que mathématique ${ }^{7}$ (fig. 1). Dès la Théorie de l'ornement, les chapitres VII à XIII sont centrés sur les figures polygonales auxquelles l'auteur accorde un statut particulier:

Il est donc utile d'examiner ces figures [à-plats polygonaux ou déterminés par des contours rectilignes] dont le caractère frappant et nettement déterminé peut aider à mieux comprendre les formes curvilignes et arrondies qui sont de composition moins simple et d'une analyse plus fine et plus profonde ${ }^{8}$.

\section{J. Bourgoin, « Polygones étoilés », p. 144 de la Théorie de l'ornement, 1873.}

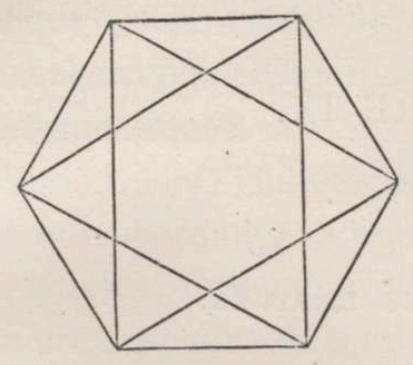

Fig. 37.

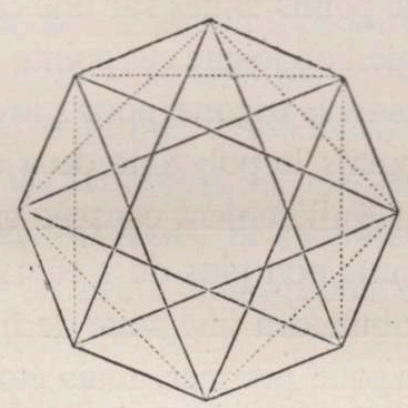

Fig. 39.

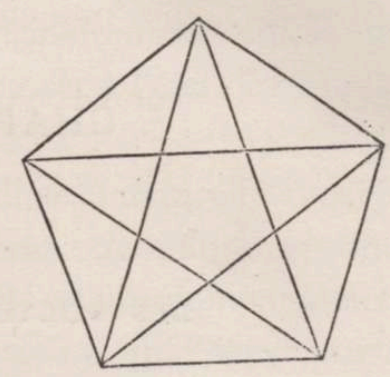

Fig. 38 .

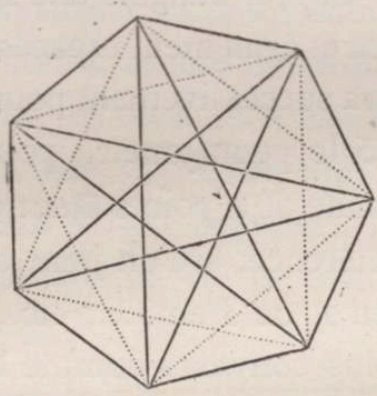

Fig. 40 .

Il énumère ensuite différents types de triangles, quadrilatères, pentagones, hexagones et s'intéresse aux possibilités de les assembler. À côté des propriétés formelles des figures polygonales, Bourgoin consacre plusieurs paragraphes à des développements 
théoriques sur les liens existants entre nombres et polygones. Il attribue à ces figures une place privilégiée dans son système, en les reliant directement à la notion d'ordre :

L'idée de nombre corrélative à l'idée de parties distinctes, et l'idée de groupement corrélative à l'idée d'un ensemble de parties, se lient immédiatement à l'idée de figure polygonale. Ces deux idées de nombre et de groupement solidaires l'une de l'autre, sont le fond purement intelligible de la notion supérieure de l'ordre, qui se lie d'autre part à la perception et à l'intelligence des formes de l'étendue?

Bourgoin a vraisemblablement sous les yeux l'ouvrage que Cournot publie en 1847 lorsqu'il rédige ces passages. En effet, quelques lignes plus loin, il paraphrase cournot en rappelant qu'« un nombre [est] une collection ou un groupe d'unités décomposable en d'autres groupes ou susceptible d'être formé par la réunion d'autres groupes ${ }^{10}$ » et il applique cette idée de groupe à la géométrie, puis à «l'architectonique » qui désigne selon lui l'ensemble des connaissances liées aux arts d'architecture et d'ornement. Ici, Bourgoin s'approprie certains aspects de la théorie de l'ordre exposée par Cournot, ce dernier s'appuyant alors sur Poinsot. Il est donc nécessaire de revenir succinctement sur les travaux de ces deux savants.

8 La division à la règle et au compas du cercle en parties égales, problème analogue à la construction géométrique des polygones réguliers, qui occupe Bourgoin dans plusieurs de ses ouvrages, constitue un exemple mathématique récurrent dans les travaux de Poinsot et de Cournot. Cette question a été traitée algébriquement par Carl Friedrich Gauss en 1801 dans son ouvrage de théorie des nombres ${ }^{11}$. En effet, les expressions trigonométriques décrivant le partage du cercle en $n$ parties égales sont les racines de l'équation binôme $x^{n}-1=0$. Gauss propose à partir de là une méthode de résolution algébrique pour obtenir des conditions de construction des polygones réguliers à la règle et au compas. Cette méthode constitue une référence fondamentale pour Poinsot, qui retient notamment le lien ainsi fait entre algèbre, théorie des nombres et géométrie.

9 Dans ses mémoires publiés entre 1808 et 1845, Poinsot souligne différentes correspondances entre ces trois domaines. Ainsi, il explicite le lien entre polygones réguliers et racines des équations binômes ${ }^{12}$ ou propose des preuves arithmétiques fondées sur des configurations géométriques ${ }^{13}$. Pour les différents cas étudiés, Poinsot met systématiquement en avant la notion d'ordre qu'il identifie à «la disposition mutuelle qu'on peut observer actuellement entre plusieurs objets ${ }^{14} »$. Dans le cas des polygones ou des racines des équations binômes, l'ordre est associé à des dispositions régulières d'objets sur un cercle, configuration ayant le rôle de modèle chez Poinsot.

10 L'ordre, associé à la situation, acquiert progressivement chez Poinsot le statut de catégorie fondamentale des mathématiques à côté de la grandeur, associée à la quantité. L'ordre permet ainsi d'atteindre "le plus haut point d'abstraction et de généralité où il soit permis de porter la science». Poinsot propose en 1845 une classification bipolaire des mathématiques qui le conduit à diviser l'algèbre, la théorie des nombres, la géométrie et la mécanique en fonction de ces deux catégories. Ainsi, il distingue l'algèbre ordinaire et l'algèbre supérieure :

Mais il y a une algèbre supérieure, qui repose tout entière sur la théorie de l'ordre et des combinaisons, qui s'occupe de la nature et de la composition des formules considérées en elles-mêmes, comme de purs symboles, et sans aucune idée de valeur ou de quantité ${ }^{15}$.

11 L'importance accordée ici aux notions d'ordre et de combinaison se retrouve dans les systèmes respectifs de Cournot et Bourgoin. 

Dès le premier chapitre, il énonce la définition abstraite d'un nombre, reprise ensuite par Bourgoin, tout en affirmant plus généralement :

Pour mettre de l'ordre dans tout assemblage d'objets individuels, pour les classer d'après leurs rapports naturels ou d'après certaines vues de notre esprit, l'on est toujours conduit à former d'abord certains groupes d'individus ou d'unités, puis à former avec ces premiers groupes des groupes supérieurs ou de second ordre ${ }^{16}$.

Ces questions de classification des objets traversent les écrits de Bourgoin. Il reprend ainsi la formulation de Cournot qu'il applique à la géométrie puis à l'architectonique et il les met en œuvre dans sa présentation des figures polygonales.

Cournot rappelle les raisonnements arithmétiques de Poinsot fondés sur des considérations graphiques. Il revient en outre sur les liens entre nombres, racines des équations binômes et polygones réguliers dans le cadre de ce qu'il appelle l'ordre périodique ${ }^{17}$. Cette dénomination particulière annonce une utilisation généralisée de la notion d'ordre par rapport à Poinsot : s'il reprend la définition que donne ce dernier de l'algèbre, Cournot propose également un statut bien plus général à la notion d'ordre, qui est alors considérée comme condition à nos connaissances.

Dans les chapitres que Bourgoin consacre aux figures polygonales transparaît à plusieurs reprises une vive tension entre son intérêt pour les mathématiques et la distance qu'il maintient fermement entre géométrie et art ornemental. Immédiatement après avoir écrit que l'on pourrait s'« étendre sur les relations curieuses qui existent entre la théorie des nombres, l'algèbre et la géométrie ${ }^{18} »$ il met en garde son lecteur : «on abandonnera aux mathématiciens, ce qui [...] relève de la curiosité scientifique ». Ainsi, il ne subordonne en aucun cas l'ornement aux mathématiques. Néanmoins, cette remarque sur le lien entre algèbre, théorie des nombres et géométrie fait écho aux propos de Poinsot et Cournot. Bourgoin mobilise ici leurs travaux afin de légitimer théoriquement le statut privilégié des figures polygonales ainsi que l'aspect fondamental de la notion d'ordre dans son système.

\section{Ordre, forme et architectonique}

Dans sa Théorie de l'ornement, Bourgoin est également sensible à la thèse développée par Cournot en $1861^{19}$ selon laquelle l'ordre et la forme représentent des catégories constitutives de notre entendement. Le propos de Cournot présente une certaine ambiguïté puisque d'un côté, l'ordre et la forme représentent l'objet privilégié des sciences logico-mathématiques, de l'autre, elles constituent une condition de possibilité des sciences en général. Cournot propose ensuite une classification des sciences, fondée sur l'ordre et la forme, et résumée dans un tableau à double entrée ${ }^{20}$ (fig. 2). 
2. A.-A. Cournot, «L'ordre et la forme », p. 105 du t. 1 du Traité de l'enchaînement des idées..., 1861.

\begin{tabular}{|c|c|c|}
\hline \multicolumn{3}{|c|}{ L'ORDRE ET LA FORME. } \\
\hline \multicolumn{2}{|c|}{ ORDRE PUREMENT INTELLIGIBLE. } & \multirow{2}{*}{$\begin{array}{l}\text { ORDRE PHÉNOMÉNAL. } \\
\text { THÉMATIQUES. }\end{array}$} \\
\hline SCIENCES LOGIQUBS. & SCIENCES MA? & \\
\hline $\begin{array}{l}\text { Idées degenre et d'espèce. } \\
\text { Classification. } \\
\text { (Syllogistique.) } \\
\text { Combinaison } \\
\text { (Syntactique.) }\end{array}$ & $\begin{array}{l}\text { Idées de nombre, } \\
\text { de rapport, } \\
\text { de grandeur et de quan- } \\
\text { tité, } \\
\text { de mesure. } \\
\text { (Théorie des nombres.- } \\
\text { Arithmétique propre- } \\
\text { ment dite. - Analyss } \\
\text { algébrique, ou théorie } \\
\text { des quantités en géné- } \\
\text { ral.). }\end{array}$ & $\begin{array}{l}\text { Idées de temps et d'es- } \\
\text { pace. } \\
\text { (Géométrie pure.) } \\
\text { (Cinématique, ou théorie } \\
\text { géométrique des mou- } \\
\text { vements.) }\end{array}$ \\
\hline $\begin{array}{l}\text { Idées de la raison des } \\
\text { choses, de la loi et de } \\
\text { l'enchânement ration- } \\
\text { nel, de l'essentiel et } \\
\text { de l'accidentel. } \\
\text { (Logique supérieure.) } \\
\text { Classification rationnelle. } \\
\text { ldée de type. }\end{array}$ & $\begin{array}{l}\text { Idées de la variation des } \\
\text { grandeurs, des varia- } \\
\text { bles indépendantes et } \\
\text { des fonctions qui en dé- } \\
\text { pendent. } \\
\text { (Théorie des fonctions, } \\
\text { analyse infinitésimale.) }\end{array}$ & $\begin{array}{l}\text { Mesure du temps. } \\
\text { (Applications de la théorie } \\
\text { des fonctions et de l'a- } \\
\text { nalyse infinitésimale à } \\
\text { la geométrie et à la ci- } \\
\text { nématique.) }\end{array}$ \\
\hline $\begin{array}{l}\text { Idée de hasard ou de com- } \\
\text { binaison fortuite. } \\
\text { Probabilité philosophi- } \\
\text { que, critique, analogie, } \\
\text { induction. }\end{array}$ & $\begin{array}{l}\text { Idée de chance. } \\
\text { Probabilité mathémati- } \\
\text { que. } \\
\text { (Calcul des probabilités.) }\end{array}$ & $\begin{array}{l}\text { Applications à la distinc- } \\
\text { tion des mouvements } \\
\text { absolusetrelatifs, réels } \\
\text { et apparents. }\end{array}$ \\
\hline
\end{tabular}

Il y distingue l'ordre intelligible, indépendant de l'expérience, et l'ordre phénoménal, qui implique les idées de temps et d'espace. Il montre que les sciences logiques relèvent de l'ordre intelligible et qu'elles embrassent la syntactique ou théorie des combinaisons dont nous préciserons les contours ci-après. Les sciences mathématiques sont réparties en fonction des idées qu'elles supposent: l'arithmétique et l'analyse algébrique dépendent exclusivement des idées de nombre, de quantité et de grandeur. Elles relèvent donc de l'ordre intelligible. La géométrie suppose l'idée d'espace et la cinématique repose au surplus sur l'idée de temps. Ces deux branches appartiennent ainsi à l'ordre phénoménal. Cournot intègre à son tableau divers domaines des mathématiques et il rend compte de leurs rapports mutuels dans toute leur subtilité. Remarquons enfin que Cournot accorde une large place à la théorie des chances et des probabilités dans la troisième ligne de ce tableau. Au final, Cournot rassemble toutes les branches des mathématiques qu'il a étudiées en propre dans des ouvrages antérieurs. Bourgoin affirme d'ailleurs les avoir lus in extenso.

Dans sa Théorie de l'ornement, Bourgoin tente de transposer à l'ornement la thèse de Cournot selon laquelle l'ordre et la forme représentent deux catégories fondamentales d'où découlent les sciences logico-mathématiques. Bourgoin est alors amené à préciser ce qu'il entend par « forme » :

L'idée usuelle de la forme correspond à toutes les formes naturelles, ouvrées ou inventionnelles, qui ont trois dimensions. Par extension, on appelle du nom de forme l'espace plan délimité, la figure ou l'à-plat terminé par des contours, et par suite ces contours eux-mêmes, c'est-à-dire les lignes ou les délinéations ${ }^{21}$.

La forme est définie à partir de ses contours dans l'espace réel ou dans celui de la page. Il ne s'agit donc pas ici d'une idée purement intellectuelle. Il souligne ensuite que la notion d'ordre ne s'identifie pas exactement à celle de forme, même si elles sont indissolublement liées. Par ordre, il entend la manière de disposer les parties d'un 
même motif ornemental ou de situer plusieurs motifs les uns par rapport aux autres. L'ordre intervient d'une part dans la composition d'une forme ornementale, en particulier lorsque celle-ci admet des propriétés de régularité et de symétrie, d'autre part dans la manière de disposer divers motifs dans un même ensemble - frises, pavages, etc.

Mais Bourgoin ne se contente pas de transposer ce cadre conceptuel à l'ornement, il s'inspire plus largement de la classification cournotienne des sciences logicomathématiques auxquelles il ajoute le domaine «architectonique ${ }^{22}$ » (fig. 3). Dans son tableau, l'architectonique n'occupe pas une position subalterne par rapport à la logique et aux mathématiques puisqu'elle s'y juxtapose. Corrélativement, il précise que la logique, les mathématiques et l'architectonique sont ordonnées de la science la plus simple à la science la plus complexe. En effet, la logique ne requiert que le langage ; l'arithmétique lui ajoute «l'algorithmie » et donc les manipulations sur des nombres; la géométrie adjoint le trait à ces deux premiers instruments. Pour finir, l'architectonique implique l'usage des quatre instruments - langage, algorithmie, trait et dessin - inscrits dans le tableau de Bourgoin.

\section{J. Bourgoin, « L'ordre et la forme » d'après Cournot, p. 24 de la Théorie de l'ornement, 1873.}

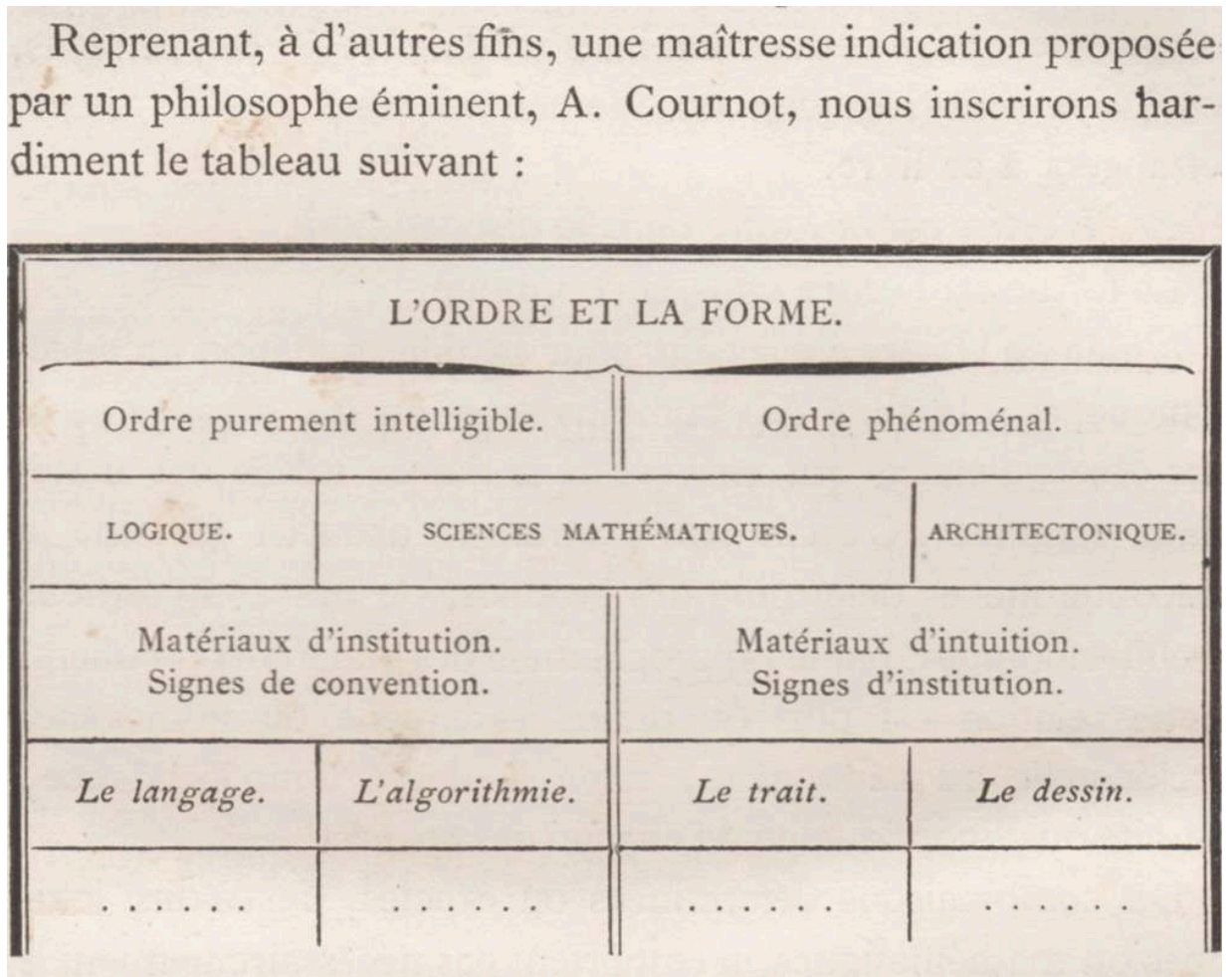

Dans ses Études architectoniques, Bourgoin revient sur cette classification et fait remarquer que Cournot accorde une place de choix à l'architectonique dans son dernier ouvrage publié en $1875^{23}$. Mais Cournot définit ce concept en un sens dérivé de Kant et assez différent de celui de Bourgoin, puisqu'il renvoie à un principe de coordination entre chaque science dans un système de connaissances. Dans le même ouvrage, Bourgoin approfondit en outre sa classification des sciences en un autre tableau très ambitieux ${ }^{24}$ qui part des trois facultés, l'intuition, la raison et l'intelligence, 
pour embrasser les sciences logiques, les arts, la psychologie, la linguistique, la politique, l'économie, la technologie, etc. (fig. 4).

4. J. Bourgoin, « L'Intuition - La Raison - L'Intelligence », p. 34 du t. 1 des Études architectoniques et graphiques, 1899.

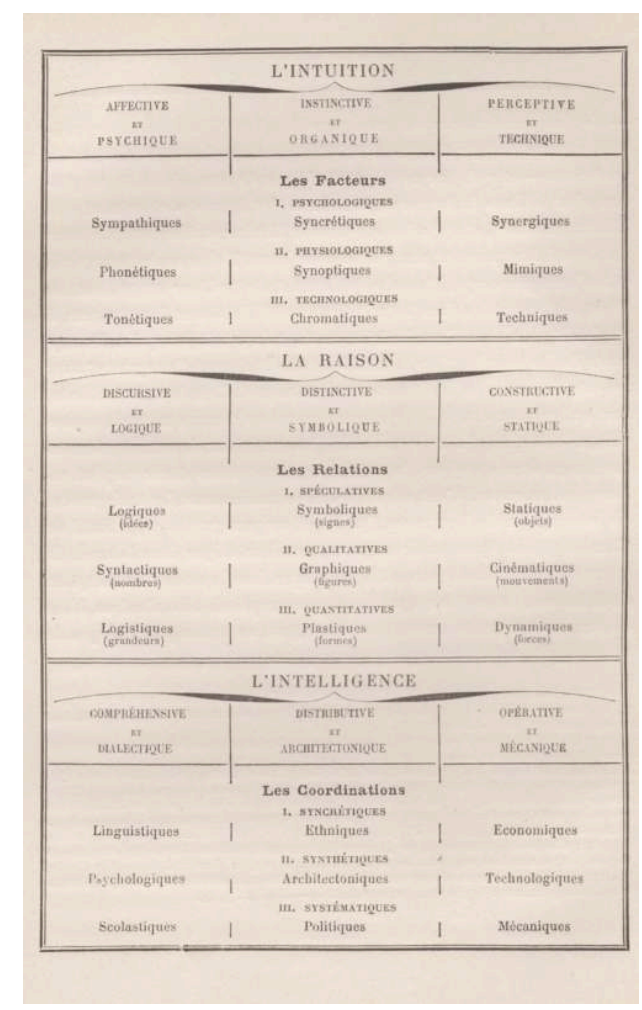

\section{Syntactique et combinaisons de motifs ornementaux}

Le chapitre premier de l'Exposition de la théorie des chances et des probabilités (1843) de Cournot est intitulé «des combinaisons et de l'ordre ». Il y évoque des résultats classiques de dénombrement, en particulier les formules permettant de calculer le nombre de combinaisons et de combinaisons ordonnées ou arrangements de $k$ objets parmi $n$. Ces résultats sont bien connus de Bourgoin qui, dans sa jeunesse, a étudié les combinaisons, les arrangements, en particulier les permutations avant d'aborder la formule du binôme de Newton $^{25}$. Il dispose donc des ressources mathématiques nécessaires pour comprendre in extenso ce chapitre de Cournot, en particulier la distinction entre une combinaison ordonnée et une combinaison, selon que l'ordre entre les objets importe ou non.

Bourgoin transpose cette distinction - qui relève initialement de la combinatoire - à l'ornement. Ceci implique de passer en revue et de classer toutes les façons de combiner et d'ordonner des motifs élémentaires. Ceci lui paraît d'autant plus justifié que Cournot ne restreint pas sa syntactique - ou théorie des combinaisons - à des questions mathématiques de dénombrement:

Parmi les idées abstraites que l'esprit humain ne crée pas arbitrairement, mais que

la nature même des choses lui suggère, l'idée de combinaison est l'une des plus générales et des plus simples. Après avoir considéré isolément des objets individuels, on est amené à concevoir que ces objets, suivant leur nature, se 
combinent ou se groupent un à un, deux à deux, trois à trois, etc., pour former certains systèmes ou objets complexes, qui peuvent se combiner à leur tour pour former d'autres groupes ou systèmes plus composés, et ainsi de suite.

La théorie des combinaisons, à laquelle les Allemands ont donné le nom de Syntactique, est une science abstraite et purement formelle, comme la science des nombres et la géométrie ${ }^{26}$. qu'il vient d'énumérer : il étudie donc bien des combinaisons ordonnées. Il se garde cependant de réduire l'art ornemental à cette approche qu'il juge "mécanique ". Bourgoin ne considère pas sa grammaire de l'ornement comme une simple ramification de la syntactique cournotienne. Reste que dans cette quatrième partie, il introduit trois types de traits, qu'il appelle "éléments linéaires »: "la droite à symétrie écartelée, l'arc à symétrie paire, et la recourbée asymétrique ou impaire ${ }^{32} »$. Il codifie chaque trait 
par une lettre minuscule et il montre ainsi comment passer en revue l'ensemble des combinaisons possibles de tels traits en permutant des lettres. Ce faisant, il transpose à l'art ornemental une manière de représenter les combinaisons qu'il avait déjà rencontrée au début de l'ouvrage de Cournot de 1843.

Bourgoin ne se contente donc pas de reprendre les arguments généraux de Cournot sur la syntactique, il est également sensible à un type d'écriture symbolique des combinaisons en mathématiques qu'il sait transformer pour l'adapter à des combinaisons d'ornements. Il refuse cependant que l'art ornemental dépende exclusivement d'une combinatoire, ceci au nom d'une thèse générale qu'il défend à maintes reprises dans la Théorie de l'ornement : les arts graphiques sont irréductibles aux sciences logico-mathématiques. La syntactique de Cournot n'est donc pas un principe directeur de l'art ornemental pour Bourgoin. Elle n'en joue pas moins un rôle essentiel dans son projet de grammaire de l'ornement.

\section{Une première application de la théorie de l'ordre à l'ornement : les « dispositions sériées »}

31 Au début de la troisième partie de sa Théorie de l'ornement, Bourgoin étudie les frises d'ornements, qu'il appelle les dispositions sériées, c'est-à-dire les séries de motifs rangés le long d'une ligne, qu'il suppose droite par commodité. Bourgoin précise que :

Les dispositions sériées, ou les rangées, sont obtenues par la répétition ou la succession alignée d'un nombre indéfini de motifs, ces motifs étant identiques ou différents les uns des autres. L'ordre et par suite le rythme s'introduisent dans les dispositions sériées par l'intervention de l'un des cinq modes fondamentaux d'arrangements: la répétition, l'alternance, l'intercalence, la période et la récurrence ${ }^{33}$.

Le terme d'arrangements suggère que l'auteur transpose aux dispositions sériées un cadre conceptuel et une symbolisation empruntés à la combinatoire. Il dégage cinq types d'arrangements remarquables par leur régularité ou leur symétrie. La répétition et l'alternance représentent les cas les plus élémentaires d'arrangement à partir de deux motifs distincts. L' 'intercalence » - le néologisme est de Bourgoin - consiste à répéter une ou plusieurs fois alternativement deux motifs distincts. Le nombre de répétitions reste fixe pour chacun des deux motifs, ce que Bourgoin illustre par une série de schémas (fig. 5 et 6 ).

5. J. Bourgoin, «Intercalence » et « Récurrence », p. 219 et 220 de la Théorie de l'ornement, 1873.

0000000000000000000000000000000

0000000000000000000000000000000

0000000000000000000000000000000

000000000000000000000000000000

Fig. 102. 


\section{0}

Fig. 104.

La période consiste à répéter un même groupe de motifs deux à deux distincts en respectant leur ordre. L'alternance est donc un cas particulier remarquable d'une période réduite à deux éléments. La récurrence consiste enfin à complexifier l'« intercalence », en y combinant plus de deux motifs. À la différence de la période, la récurrence implique d'introduire des propriétés de symétrie.

La récurrence induit le plus de complexité et de variété. Pour l'appréhender, Bourgoin associe une lettre minuscule à chacun des motifs pour ensuite les combiner. Il emprunte une nouvelle fois cette codification à un type d'écriture bien connu en dénombrement.

Soulignons que les idées de régularité, de variété et de symétrie jouent un rôle cardinal dans cette classification des dispositions sériées. La syntactique de Cournot n'est pas la seule référence mobilisée pour aborder les arrangements. Lors de ses investigations en bibliothèque, Bourgoin découvre les deux Essais de taxéométrie du mathématicien et philosophe Johann-Heinrich Lambert, publiés respectivement en 1770 et en $1773^{34}$, dans lesquels la notion d'ordre est étudiée d'un point de vue tant métaphysique, mathématique qu'esthétique (fig. 7). Dans le premier Essai qui n'intéresse Bourgoin qu'indirectement, Lambert complète la définition, établie par Christian Wolff, de l'ordre comme "ressemblance de ce qui est simultané et successif ${ }^{35}$ ». Selon Lambert, Wolff définit ici l'ordre local ou ordre de ressemblance comme arrangement des parties d'un objet ou de plusieurs objets selon des principes de symétrie. Lambert affirme que «l'ordre le plus simple c'est l'ordre linéaire, en ce qu'il n'a qu'une seule dimension locale ${ }^{36} »$. Lambert classe donc divers types d'ordres (locaux) en fonction du nombre de dimensions qu'ils impliquent. De manière similaire, Bourgoin commence par les frises ordre unidimensionnel - pour ensuite aborder les réseaux et pavages du plan - ordre bidimensionnel. Il est frappant de constater que Lambert, comme le fera Bourgoin, illustre son propos sur l'ordre linéaire en se référant à des objets de nature différente éléments d'architecture, langage, musique : « Telle est, par exemple, une suite d'arbres qui bordent une allée, telle est une suite de colonnes ou d'arcs qui soutiennent un aqueduc. Telle est la mélodie d'un air qu'on chante, et tel est encore chaque discours qu'on prononce $^{37}$.» 


\section{J. Bourgoin, Notes d'après le « Second essai de taxéométrie » de Johann Heinrich Lambert.}

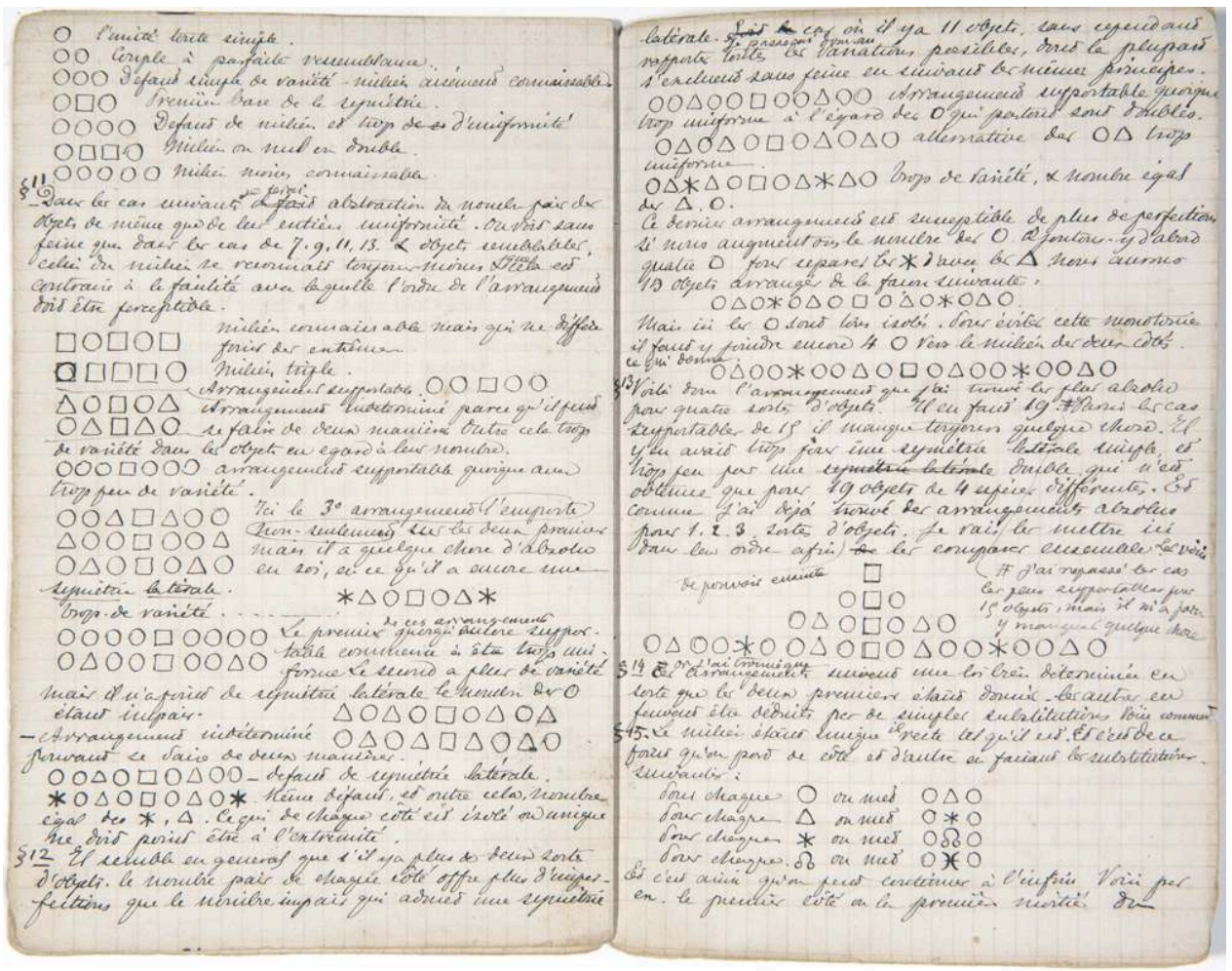

Source: Paris (France), Bibliothèque de I'Institut national d'histoire de l'art, collections Jacques Doucet, Arch. 67, 11, 4

Lambert entend ensuite mesurer et calculer le degré d'ordre dans un arrangement d'un nombre fixé d'objets. Ces considérations mathématiques, que Bourgoin ne reproduit pas dans ses notes, justifient le sous-titre de l'Essai de Lambert: «sur la mesure de l'ordre ».

Dans le second essai, Lambert s'intéresse plus spécifiquement aux effets esthétiques de certains arrangements linéaires de formes géométriques élémentaires en fonction de trois critères à équilibrer : régularité, symétrie et variété. Or, on retrouve ces trois critères dans la classification des dispositions sériées de Bourgoin.

Notons que Lambert recherche un juste milieu entre la monotonie et la trop grande variété. En outre, il repère les arrangements «indéterminés » qui, à une permutation près, contribuent aux mêmes effets esthétiques. Enfin, Lambert suggère que les arrangements sont d'autant plus appréciables qu'ils impliquent de combiner plusieurs symétries. Le second Essai de Lambert intéresse Bourgoin à trois niveaux. Tout d'abord Lambert étudie l'ordre comme arrangement entre des objets d'un point de vue esthétique, ce que ne faisaient ni Poinsot, ni Cournot. Ensuite, Lambert décrit des arrangements que Bourgoin décrit sous le terme de récurrence. Enfin et surtout, Lambert utilise un type de notation par figures géométriques élémentaires analogue à celui que l'on retrouve dans certains carnets de Bourgoin ${ }^{38}$ (fig. 8). 


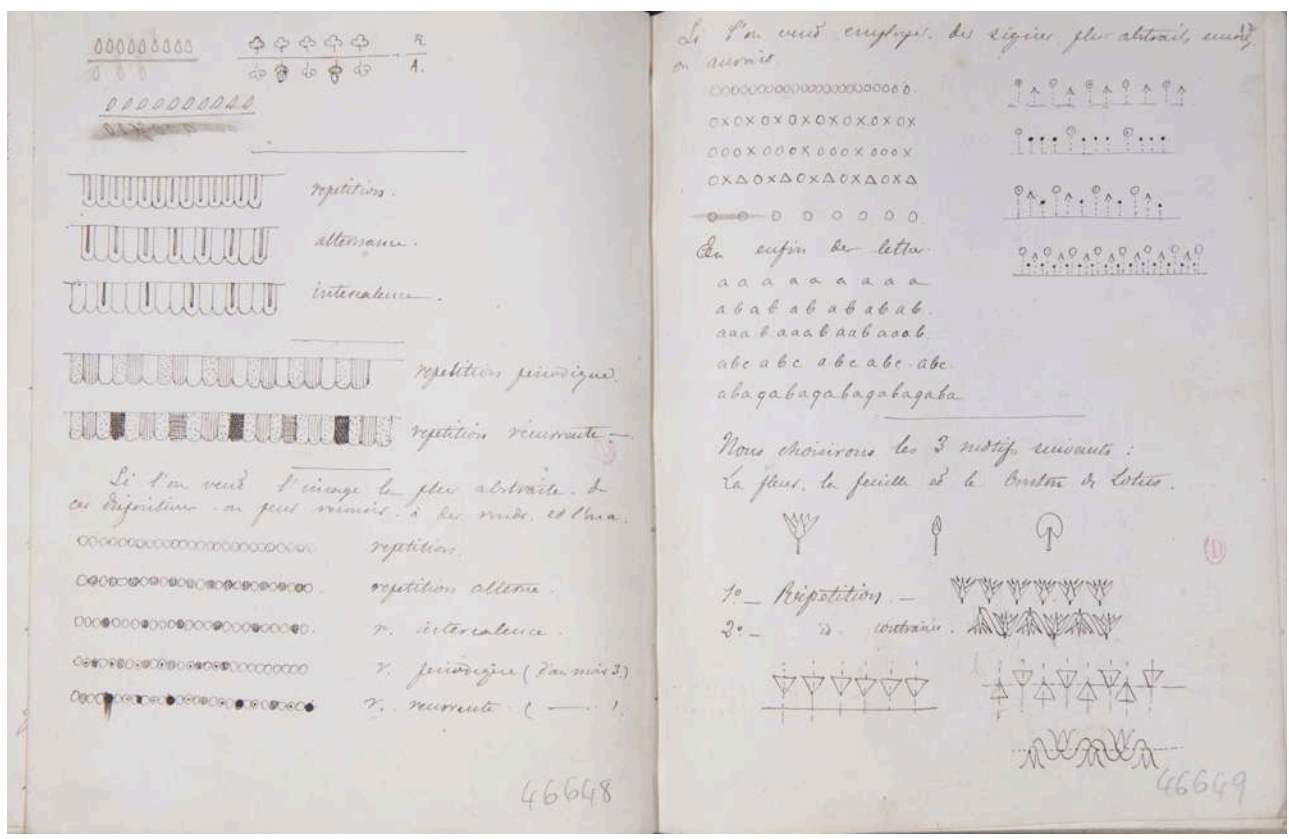

Source: Paris (France), Bibliothèque de l'Institut national d'histoire de l'art, collections Jacques Doucet, Arch. 67, 10, 5.

Ce dernier expérimente différents modes de notation pour les dispositions sériées : 1 . à l'aide d'une succession de petits cercles blancs, noirs ou avec un point ou une autre figure en leurs centres, 2. par une suite de lettres minuscules à l'image des arrangements en combinatoire, - ces deux types de représentation sont utilisés dans sa Théorie de l'ornement, - 3. par des figures géométriques élémentaires - cercles, triangles, étoiles -, à l'instar de Lambert dans ses Essais de taxéométrie. Ces éléments démontrent que le corpus de Bourgoin sur la théorie de l'ordre n'est pas restreint à Cournot et Poinsot. En outre, l'exemple des frises ou des dispositions sériées montre que Bourgoin sait transformer ses connaissances en combinatoire pour qu'elles répondent à des préoccupations esthétiques et artistiques. Le second Essai de taxéométrie de Lambert constitue justement un point de jonction remarquable entre les mathématiques et l'esthétique sur le thème des arrangements, d'où l'exemplarité de ce texte que Bourgoin n'a pas hésité à prendre en note in extenso.

\section{Une deuxième application : armures textiles et échiquiers}

Les figures textiles traversent l'œuvre de Bourgoin de 1873 à 1905. Elles constituent un corpus privilégié pour l'étude des dispositions des traits et elles représentent l'une des principales applications de sa science de la "graphique ${ }^{39}$ ». Il s'intéresse aux formes textiles dès la Théorie de l'ornement, dans la troisième partie consacrée à «L'ordre et la disposition dans l'ornement et les formes ». La deuxième section est composée d'un développement théorique sur les réseaux et les groupements de cercles. Bourgoin souligne ensuite leurs usages possibles : "Ces notions théoriques et très générales une fois établies, leur application à l'ornementation en découle aussitôt $t^{40} \%$. Les arts textiles sont alors évoqués comme une application importante des réseaux quadrillés. Dans un 
court paragraphe, Bourgoin parle de "contre-semplage" et insère des figures semblables à celles que l'on trouve dans le Traité encyclopédique et méthodique de la fabrication des tissus de Pierre Falcot publié en $1852^{41}$ (fig. 9 et 10).

\section{J. Bourgoin, « Contre-semplages », p. 267 de la Théorie de l'ornement, 1873.}

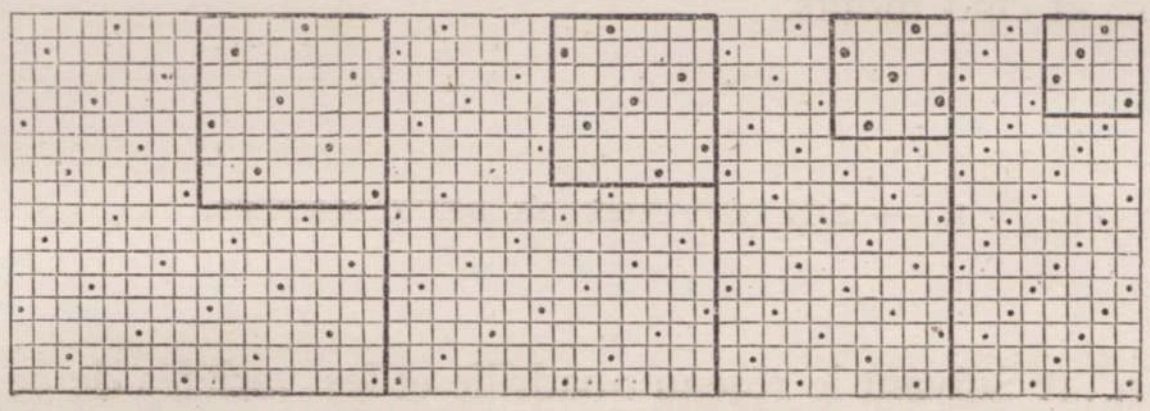

Fig. $2 \mathrm{I} 4$.

10. P. Falcot, pl. 40 du Traité encyclopédique et méthodique de la fabrication des tissus, 1852.

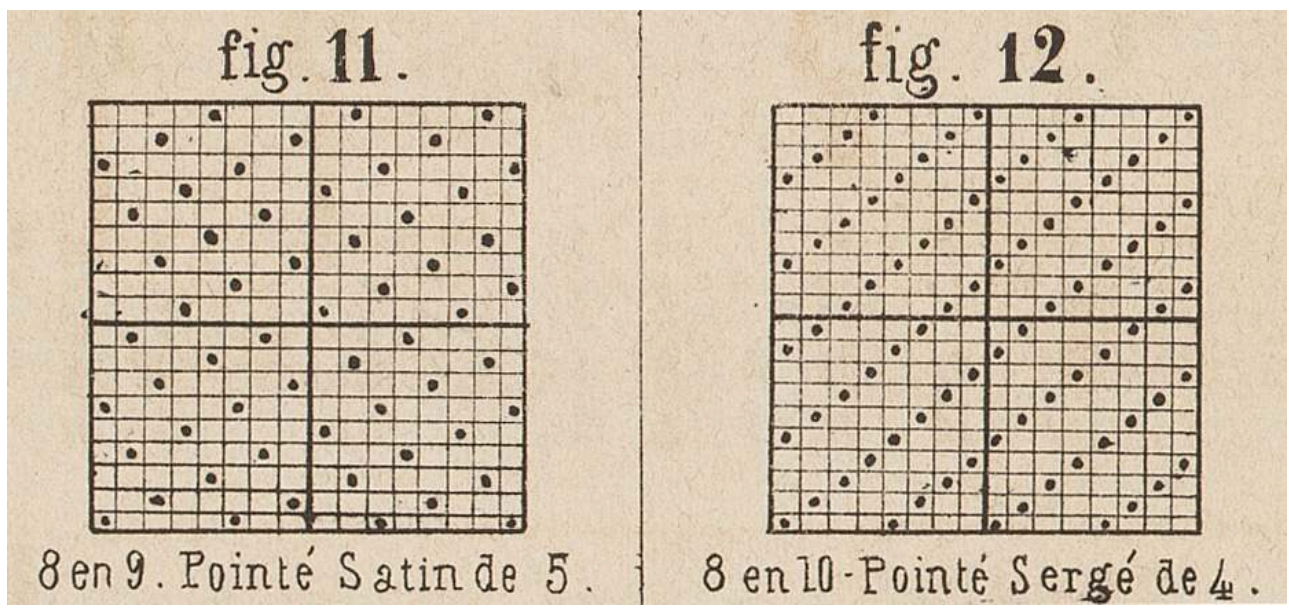

41 Évoquées dans le chapitre «les nœuds et les tresses » de la Grammaire élémentaire de l'ornement ${ }^{42}$, les figures textiles continuent d'être exploitées par Bourgoin comme une application de sa "graphique » entre 1901 et 1905. Elles sont le sujet de nombreuses planches du second volume des Études architectoniques et graphiques, symbolisées à l'aide de réseaux quadrillés. Les arts textiles sont explicitement mentionnés dans le troisième tome de la Graphique, « Développements et applications ». Par exemple, les figures 11 et 12 de la planche 58 (fig. 11) correspondent respectivement à un motif tissé et à son interprétation comme un bref d'armure sous la forme d'un échiquier, ce qui est courant dans les arts textiles. 

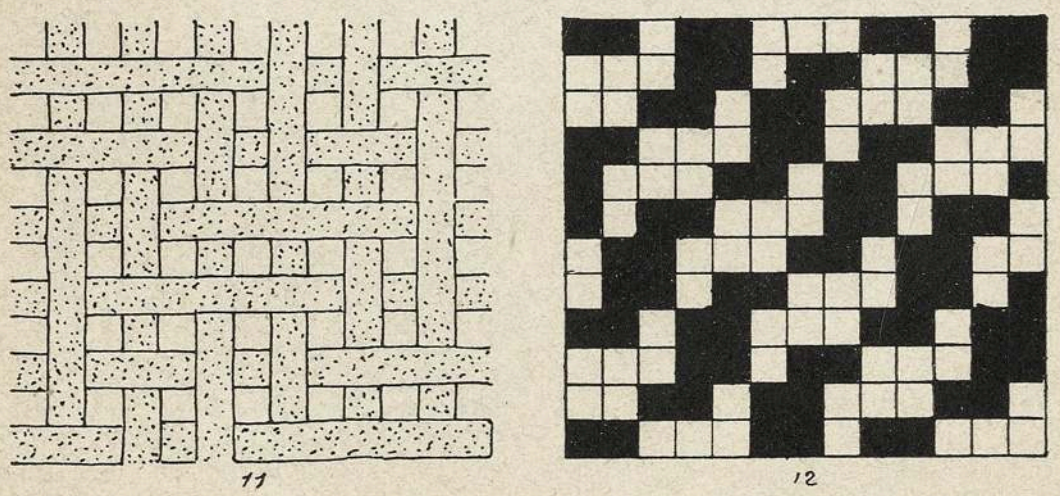

Le deuxième tome de La Graphique, justement consacré aux échiquierss ${ }^{43}$, témoigne à nouveau de sa grande familiarité avec le vocabulaire de l'analyse combinatoire. Il y évoque différents types de permutations; il se réfère à plusieurs reprises aux géomètres ainsi qu'aux mathématiques et il traite analytiquement différents échiquiers carrés de $4,9,16$ et 25 cases en fonction des conditions qui président au placement des cases noires et blanches.

Dans ses derniers ouvrages, Bourgoin continue de développer son système de notation en revenant sur l'importance des notions d'ordre et de forme en lien avec les figures textiles : « Nous sommes ainsi amenés à envisager les problèmes relatifs à l'ordre et à la situation, à la disposition et à la forme, sous deux aspects principaux ${ }^{44} »$ : les questions en lien avec le nombre et celles en lien avec l'étendue. Pour illustrer son propos, Bourgoin reproduit des extraits d'un mémoire d'Alexandre Théophile Vandermonde sur les problèmes de situation ${ }^{45}$ inséré dans les Mémoires de l'Académie des sciences de Paris. Dans ce texte, régulièrement cité au XIX ${ }^{e}$ siècle lorsqu'il est question de géométrie de situation, Vandermonde se réfère principalement aux entrelacements de fils et au problème du cavalier dans les jeux d'échecs. Bourgoin ne retient de ce texte que les extraits qui servent sa théorie et estime que Vandermonde exprime "par un système de notation à la fois syntactique et géométrique, les idées fondamentales de l'ordre et de la forme ${ }^{46} »$.

Bourgoin s'est également constitué un corpus de références autour des satins, incluant un Traité de tissage de Théodore Bona (1863) ${ }^{47}$, dont il étudie les brefs d'armure (fig. 12), ainsi que les «nouvelles méthodes de construction des satins ${ }^{48}$ » d'Édouard Gand qui ouvrent vers des approches mathématiques (fig. 13). Ce professionnel du textile, enseignant en école technique, souhaite en effet déterminer des " procédés rationnels " permettant d'obtenir sans tâtonnements la "mise en carte du satin proposé»; préoccupations auxquelles répondent certaines recherches de l'arithméticien Édouard Lucas sur la " géométrie des tissus ${ }^{49}$ ». 
12. J. Bourgoin, Diagrammes de satin d'après Th. Bona, Traité de tissage, 1863.

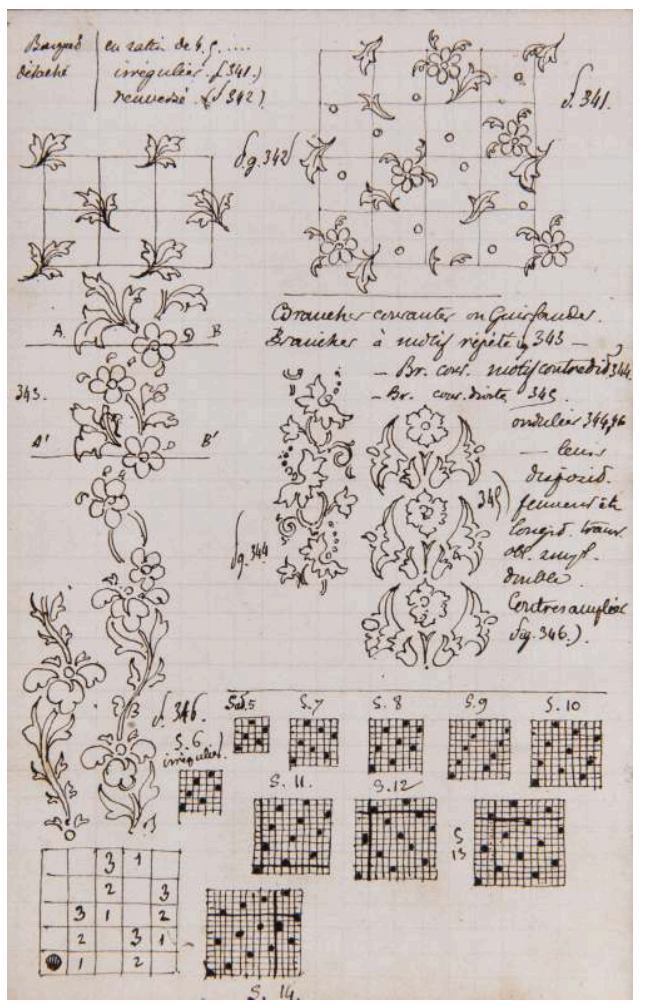

Source : Paris (France), Bibliothèque de l'Institut national d'histoire de l'art, collections Jacques Doucet, Arch. 67, 7, 2.

13. É. Gand, Diagramme de satin, p. 47 du t. 1 du Cours de tissage en soixante-quinze leçons, $3^{\mathrm{e}}$ éd., 1886.

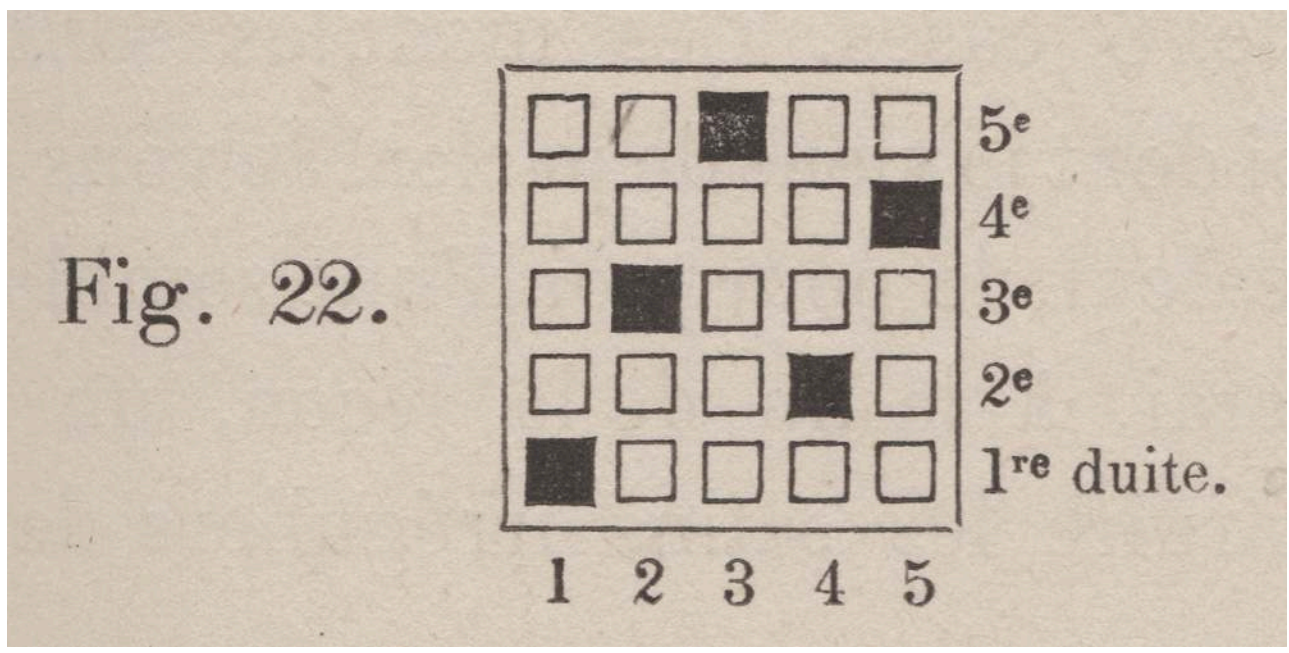

La place fondamentale que prennent les « figures d'échiquiers » dans La Graphique doit ainsi être comprise au regard des travaux de mathématiciens contemporains. À partir des années 1870, Édouard Lucas, Charles-Ange Laisant ou encore Eugène Catalan développent des pratiques mathématiques au croisement entre la théorie des nombres, la combinatoire et la géométrie de situation. Membres de la Société mathématique de France, ces mathématiciens développent également leurs travaux au sein de l'Association française pour l'avancement des sciences. Dans ce cadre où les 
mathématiques utiles sont mises en avant, les représentations visuelles, parmi lesquelles les échiquiers, sont privilégiées pour traiter certains problèmes et vulgariser les connaissances.

L'auteur le plus prolifique sur ces questions est Lucas. Dans sa Théorie des nombres ${ }^{50}$, publiée l'année de sa mort en 1891, Lucas s'intéresse à la combinatoire (chapitre VI) et à la géométrie de situation (chapitre VII), étudiant alors consécutivement les échiquiers, les polygones, les réseaux, les régions et les polyèdres. S'il ne le cite pas, c'est à Lucas que Bourgoin fait implicitement référence lorsque, dans le tome II de La Graphique consacré aux "figures d'échiquiers", il évoque "ce que les géomètres appellent les permutations figurées", à savoir des échiquiers "qui ne contiennent qu'un seul point noir sur une même ligne, colonne ou rangée $e^{51}$ ", situation qui correspond en fait aux satins réguliers. De plus, la planche 61 de Bourgoin est quasiment identique à la figure 28 issue de la Théorie des nombres de Lucas (fig. 14 et 15). L'intérêt de Bourgoin pour la combinatoire et la géométrie de situation l'a vraisemblablement conduit à consulter les travaux de Lucas. Ceci montre une fois de plus que Bourgoin s'appuie, jusqu'à la fin de sa carrière, sur des ouvrages de mathématiques théoriques pour alimenter sa science de l'ornement. Remarquons enfin que Lucas, dans l'introduction de sa Théorie des nombres, renvoie à Poinsot lorsqu'il insiste sur l'importance de la notion d'ordre dans la science des nombres ${ }^{52}$.

14. J. Bourgoin, « Échiquiers », p. 61 du t. 2 de La Graphique, 1905.

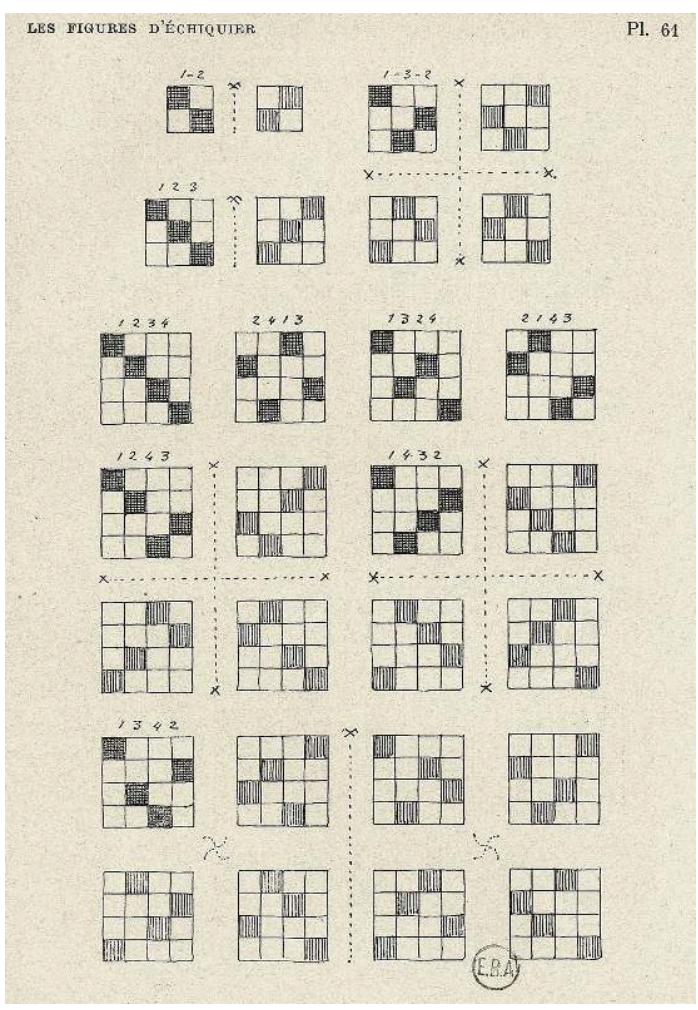


15. É. Lucas, «Permutations figurées de quatre éléments », p. 66 du t. 1 de la Théorie des nombres, 1891.

Fig. 28 .
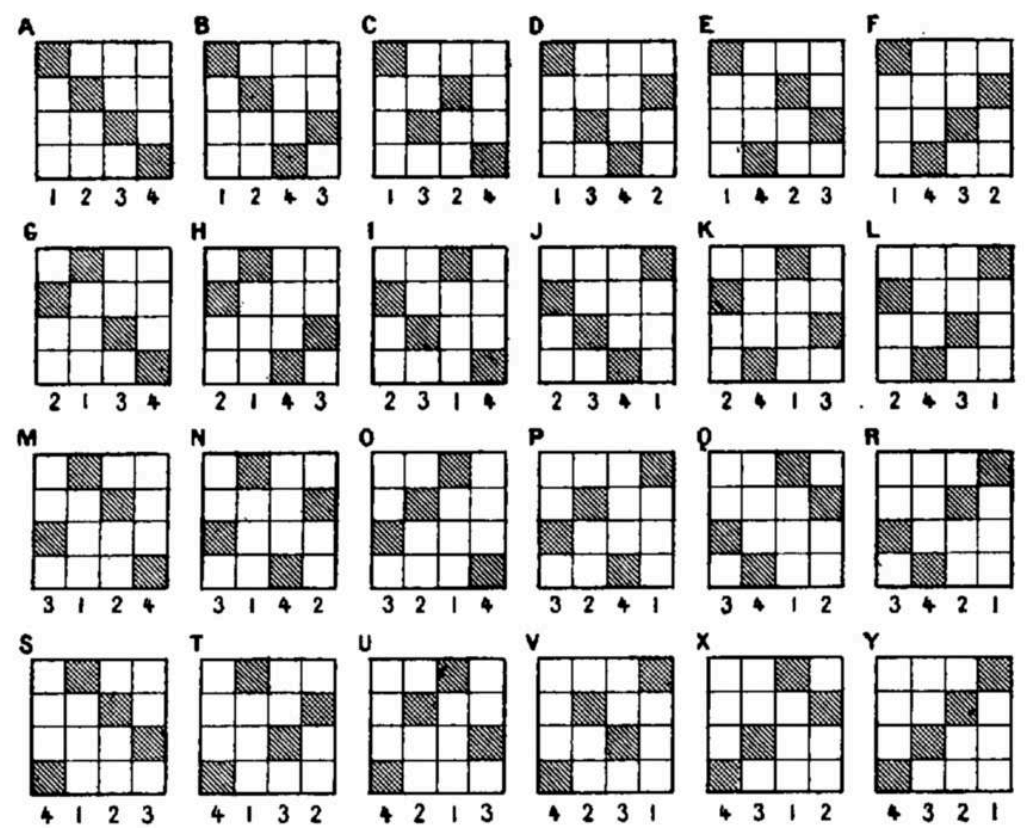

Les permutations figurées de quatre éléments.

La syntactique et la théorie de l'ordre de Cournot constituent l'une des principales sources d'inspiration de Bourgoin dès sa Théorie de l'ornement. Il s'approprie cet héritage cournotien à trois niveaux : les figures polygones, centrales dans l'art ornemental ; la classification des sciences logico-mathématiques, auxquelles Bourgoin ajoute l'architectonique; l'étude des combinaisons ordonnées que Bourgoin utilise pour classer des manières de disposer des motifs ornementaux. À partir de sa lecture de Cournot, Bourgoin remonte à la théorie de l'ordre telle qu'elle est thématisée par Poinsot dans le premier XIX ${ }^{\mathrm{e}}$ siècle. En outre, Bourgoin enrichit son corpus sur l'ordre lorsqu'il étudie les frises ou dispositions sériées, ainsi que les figures d'échiquiers. En effet, les notes de Bourgoin attestent de son intérêt pour le second Essai de taxéométrie de Lambert. Ce dernier classe alors des arrangements de figures géométriques élémentaires en fonction de leurs effets esthétiques. Plusieurs de ces arrangements font penser aux dispositions sériées de Bourgoin. Enfin, dans le cas des figures d'échiquiers, Bourgoin s'appuie tout d'abord sur le mathématicien Vandermonde qui fait le lien entre géométrie de situation et arts du textile. Bourgoin partage en outre une référence commune avec les mathématiciens Laisant et Lucas, à savoir Gand. Plus profondément, il apparaît que Bourgoin a consulté le traité de théorie des nombres de Lucas, ce qui renforce ses liens avec certains mathématiciens qui, dès les années 1870 , rendent plus explicites les applications de la théorie des nombres, de la combinatoire et de la géométrie de situation à l'industrie textile. Mais si ces protagonistes semblent sensibles à une rationalisation voire à une mathématisation de ce secteur industriel, Bourgoin, dans tous ses ouvrages y compris $L a$ Graphique, refuse de définir l'art ornemental comme un simple prolongement de la syntactique cournotienne entendue comme théorie des combinaisons. Bien que séduit par cette théorie, il la tient à distance de l'art ornemental. 


\section{NOTES}

1. Pour une analyse plus poussée des liens entre les ouvrages de Cournot et de Bourgoin, voir Jenny BOUCARD et Christophe ECKES, «La théorie de l'ordre de Poinsot et Bourgoin: mathématiques, philosophie, art monumental ", Revue de synthèse, vol. 136, nº 3-4, déc. 2015 (Le vif de l'histoire), p. 403-447.

2. Jules Bourgoin, Études architectoniques et graphiques, t. 1, Paris : Ch. Schmid, 1899, p. 26.

3. Antoine-Augustin COURNOT, Traité de l'enchaînement des idées fondamentales dans les sciences et dans l'histoire, Paris : Hachette, 1861.

4. Jules BOURGOIN, «L'architectonique ", manuscrit préparatoire pour les Études architectoniques, Paris (France), Bibliothèque de l'Institut d'histoire de l'art, collections Jacques Doucet, Arch. 67, 5, 5.

5. Antoine-Augustin COURNOT, De l'origine et des limites de la correspondance entre l'algèbre et la géométrie, Paris : Hachette, 1847.

6. ID., Exposition de la théorie des chances et des probabilités, Paris : Hachette, 1843.

7. Pour les sources de cette réflexion, voir Jenny BOUCARD et Christophe ECKES, «Les sources scientifiques de Jules Bourgoin : cristaux, polygones et polyèdres », supra, p. 299-318.

8. Jules BOURGoIn, Théorie de l'ornement, Paris : A. Lévy, 1873, p. 117.

9. Ibid., p. 118.

10. Ibid. Voir Antoine-Augustin COURNOT, De l'origine et des limites..., op. cit. (note 5), p. 3.

11. Carl Friedrich GAUSS, Disquisitiones Arithmeticae, Leipzig: Fleischer, 1801. Voir Catherine GOLDSTEIN, Norbert SCHAPPACHER et Joachim SCHWERMER (dirs.), The Shaping of Arithmetic after C. F. Gauss's Disquisitiones Arithmeticae, Berlin : Springer, 2007 et Jenny BOUCARD, « Louis Poinsot et la théorie de l'ordre: un chaînon manquant entre Gauss et Galois?», Revue d'histoire des mathématiques, vol. 17, $\mathrm{n}^{\circ} 1,2011$, p. 41-138.

12. Louis POINSOT, «Mémoire sur les polygones et les polyèdres ", Journal de l'École polytechnique, vol. $4,10^{\mathrm{e}}$ cahier, 1810 , p. 16-49.

13. ID., «Réflexions sur les principes fondamentaux de la théorie des nombres ", Journal de mathématiques pures et appliquées, vol. 10, 1845, p. 1-101.

14. ID., «Mémoire sur l'application de l'algèbre à la théorie des nombres ", Journal de l'École polytechnique, vol. 11, 1820, p. 402.

15. ID., « Réflexions sur les principes fondamentaux de la théorie des nombres », op. cit., p. 4.

16. Antoine-Augustin cournot, De l'origine et des limites..., op. cit. (note 5), p. 6.

17. Ibid., chapitre XII, p. 255-256.

18. Jules BOURGoIn, Théorie..., op. cit. (note 8), p. 157.

19. Antoine-Augustin CoURNoT, Traité de l'enchaînement des idées..., op. cit. (note 3).

20. Ibid., p. 105, tableau « L'ordre et la forme».

21. Jules BOURGoIN, Théorie..., op. cit. (note 8), p. 19.

22. Ibid., p. 24, tableau « L'ordre et la forme » dérivé de Cournot.

23. Antoine-Augustin CouRnot, Matérialisme, vitalisme, rationalisme. Études sur l'emploi des données de la science en philosophie, Paris : Hachette, 1875, p. 369-370.

24. Jules BOURGoIN, tableau "L'Intuition - La Raison - L'Intelligence ", Études architectoniques et graphiques, t. 1, op. cit. (note 2), p. 34 .

25. Voir Jenny BOUCARD et Christophe ECKES, «Les sources scientifiques de Jules Bourgoin... », infra, p. 299-318.

26. Antoine-Augustin COURNOT, Exposition de la théorie des chances..., op. cit. (note 6), p. 1.

27. Ibid., p. 2. 
28. Thierry MARTIN, "Probabilités et philosophie des mathématiques chez Cournot", Revue d'histoire des mathématiques, vol. 1, 1995, chap. III.

29. Jules Bourgoin, Théorie..., op. cit. (note 8), p. 214. Voir aussi Estelle thiBAult, « La Grammaire élémentaire de l'ornement : modèles linguistiques et enjeux pédagogiques », supra, p. 231-254.

30. Ibid., p. 311.

31. Ibid., p. 312.

32. Ibid., p. 312.

33. Ibid., p. 219-220.

34. Johann Heinrich LAMBERT, «Essai de taxéométrie, ou sur la mesure de l'ordre ", Nouveaux mémoires de l'Académie royale des sciences et belles-lettres de Berlin, Année 1770, 1772, p. 327-342 ; ID., «Second essai de taxéométrie, ou sur la mesure de l'ordre ", Nouveaux mémoires de l'Académie royale des sciences et belles-lettres de Berlin, Année 1773, 1775, p. 347-368. Paris (France), Bibliothèque de l'Institut d'histoire de l'art, collections Jacques Doucet, Arch. 67, 11, 4.

35. ID., « Essai de taxéométrie... », op. cit. (note 34), p. 329.

36. Ibid., p. 331.

37. Ibid., p. 332.

38. Paris (France), Bibliothèque de l'Institut d'histoire de l'art, collections Jacques Doucet, Arch. 67, 10, 5 (carnet 31).

39. Voir Estelle thIBAULT, « La graphique, une science des figures pour l'école et l'atelier », supra, p. 255-280.

40. Jules BOURGoIN, Théorie..., op. cit. (note 8), p. 265.

41. Pierre falcot, Traité encyclopédique et méthodique de la fabrication des tissus, Elbeuf : l'Auteur, $2^{\mathrm{e}}$ éd., 1852, vol. 1, p. 355 (contre-semplage) et vol. 2, pl. 40. Voir Jules BOURGoIn, Théorie..., op. cit. (note 8), p. 267.

42. Jules Bourgoin, Grammaire élémentaire de l'ornement, Paris : Ch. Delagrave, 1880, p. 112-122.

43. Estelle тнівAULt, «Figures d'échiquiers", dans Jules Bourgoin 1838-1908. L'obsession du trait, catalogue d'exposition (Paris, Institut national d'histoire de l'art, 20 novembre 2012-12 janvier 2013), Paris : INHA, 2012, p. 46- 47.

44. Jules Bourgoin, Études architectoniques et graphiques, t. 2, Paris : Ch. Schmid, 1901, p. 20.

45. Alexandre-Théophile VANDERMONDE, "Remarques sur les problèmes de situation ", Histoire de l'Académie royale des sciences, avec les Mémoires de mathématiques et de physique, Année 1771, 1774, p. 566-574.

46. Jules BOURGoIN, Études architectoniques..., t. 2, op. cit. (note 44), p. 20.

47. Théodore BonA, Traité de tissage, Bruxelles : E. Tarlier, 1863. Voir INHA, Arch. 67, 7, 2.

48. Édouard GAND, « Nouvelles méthodes de construction des satins réguliers, pairs et impairs. Théorie des nombres premiers appliquée aux pointés de ces armures ", Bulletin de la Société industrielle d'Amiens, Janvier 1867, p. 57-88.

49. Édouard LUCAS, Application de l'arithmétique à la construction de l'armure des satins réguliers, Paris : G. Retaux, 1867. Voir à ce propos Anne-Marie DÉCAILLOT, «Géométrie des tissus. Mosaïques. Échiquiers. Mathématiques curieuses et utiles ", Revue d'histoire des mathématiques, vol. 8, 2002, p. 145-206.

50. ID., Théorie des Nombres, t. 1, Paris : Gauthier-Villars, 1891.

51. Jules Bourgoin, La Graphique, t. 2, Paris: Delagrave, 1905, p. 12. L'expression «permutations figurées" est utilisée dès 1883 dans Édouard LUCAS, "Sur l'arithmétique figurative - les permutations ", Association française pour l'avancement des sciences. Compte-rendu de la $12^{e}$ session. Rouen. 1883,1884 , p. 83-84.

52. Édouard LUCAS, Théorie des Nombres, op. cit. (note 50), p. XVIII. 


\section{AUTEURS}

\section{JENNY BOUCARD}

Jenny Boucard est maître de conférences en histoire des sciences à l'université de Nantes et membre du centre François-Viète. Ses travaux portent sur l'histoire de la théorie des nombres (second $\mathrm{XVIII}^{\mathrm{e}}$ siècle-XIX ${ }^{\mathrm{e}}$ siècle) et sur la théorie de l'ordre.

\section{CHRISTOPHE ECKES}

Christophe Eckes est maître de conférences en mathématiques, spécialisé en histoire des mathématiques, à l'université de Lorraine et membres des Archives Henri Poincaré. Ses travaux portent sur l'histoire de l'œuvre mathématique et philosophique de Hermann Weyl (1885-1955), l'histoire de la perspective aux XVIII ${ }^{\mathrm{e}}$ et XIX ${ }^{\mathrm{e}}$ siècles et sur l'histoire de l'art ornemental au XIX siècle. 